Homology, Homotopy and Applications, vol.5(1), 2003, pp.423-436

\title{
THE SET OF RATIONAL HOMOTOPY TYPES WITH GIVEN COHOMOLOGY ALGEBRA
}

\author{
HIROO SHIGA AND TOSHIHIRO YAMAGUCHI
}

(communicated by James Stasheff)

\begin{abstract}
For a given commutative graded algebra $A^{*}$, we study the set $\mathcal{M}_{A^{*}}=\left\{\right.$ rational homotopy type of $\left.X \mid H^{*}(X ; Q) \cong A^{*}\right\}$. For example, we see that if $A^{*}$ is isomorphic to $H^{*}\left(S^{3} \vee S^{5} \vee\right.$ $\left.S^{16} ; Q\right)$, then $\mathcal{M}_{A^{*}}$ corresponds bijectively to the orbit space $P^{3}(Q) / Q^{*} \amalg\{*\}$, where $P^{3}(Q)$ is the rational projective space of dimension 3 and the point $\{*\}$ indicates the formal space.
\end{abstract}

\section{Introduction}

For a given graded algebra over the rationals (abbreviated to G.A.) $A^{*}$, there exists at least one rational homotopy type having $A^{*}$ as a cohomology algebra, namely the formal space. In general there are many rational homotopy types having isomorphic cohomology algebras. In [5] it was shown that there are two rational homotopy types with isomorphic cohomology algebras and isomorphic homotopy Lie algebras, and in [6] it was shown that there are infinitely many rationally elliptic homotopy types having isomorphic cohomology algebras. Set

$$
\mathcal{M}_{A^{*}}=\left\{\text { rational homotopy type of } X \mid H^{*}(X ; Q) \cong A^{*}\right\} .
$$

The set $\mathcal{M}_{A^{*}}$ was studied by several authors $([\mathbf{1}],[\mathbf{2}],[\mathbf{3}],[\mathbf{7}],[\mathbf{1 0}])$. For example, Lupton $([\mathbf{3}])$ showed that for any positive integer $n$ there is a G.A. $A^{*}$ such that the cardinality of $\mathcal{M}_{A^{*}}$ is $n$. Halperin and Stasheff studied $\mathcal{M}_{A^{*}}$ by the set of perturbations of the differential of the formal differential graded algebra (abbreviated to D.G.A.). In particular they showed for $A^{*}=H^{*}\left(\left(S^{2} \vee S^{2}\right) \times S^{3} ; Q\right)$, the set $\mathcal{M}_{A^{*}}$ consists of two points. This example is also caluculated from our view point (see Section 3(4)). Schlessinger and Stasheff $([\mathbf{7}])$ extended the arguments in [2].

We study $\mathcal{M}_{A^{*}}$ from a different point of view. Our strategy to study $\mathcal{M}_{A^{*}}$ is as follows. We construct inductively 1-connected minimal algebras $m_{n-1}$ such that there is a G.A.map

$$
\sigma_{n}:\left(H^{*}\left(m_{n-1}\right)(n)\right)^{*} \rightarrow A^{*}
$$

so that $\sigma^{i}$ is isomorphic for $i \leqslant n-1$ and monomorphic for $i=n$, where $\left(H^{*}\left(m_{n-1}\right)(n)\right)^{*}$ is the sub G.A. of $H^{*}\left(m_{n-1}\right)$ generated by elements of degree

Received July 25, 2003, revised October 5, 2003; published on October 15, 2003.

2000 Mathematics Subject Classification: 55p62.

Key words and phrases: rational homotopy type, minimal algebra, $k$-intrinsically formal (k-I.F.)

(C) 2003, Hiroo Shiga and Toshihiro Yamaguchi. Permission to copy for private use granted. 
$\leqslant n$. Suppose we have constructed the pair $\left(m_{n-1}, \sigma_{n-1}\right)$. Then there is a unique minimal algebras $m_{D}$ containing $m_{n-1}$ and a G.A.map

$$
\sigma_{D}:\left(H^{*}\left(m_{D}\right)(n)\right)^{*} \rightarrow A^{*}
$$

such that $\sigma_{D}{ }^{i}$ is isomorphic for $i \leqslant n-1$, monomorphic for $i=n$ and moreover $\sigma_{D}^{n+1}$ induces an isomorphism on the decomposable part

$$
\sigma_{D}^{n+1}:\left(H^{*}\left(m_{D}\right)(n)\right)^{n+1} \rightarrow(A(n))^{n+1},
$$

where $(A(n))^{n+1}$ is the degree $n+1$ part of the subalgebra $A(n)$ of $A^{*}$ generated by elements of degree $\leqslant n$. To construct $m_{n}$ we choose a subspace $W$ of $H^{n+1}\left(m_{D}\right)$ satisfying certain conditions (see (2.3) and (2.4) in Section 2) so that $H^{n+1}\left(m_{n}\right) \oplus$ $W=H^{n+1}\left(m_{D}\right)$.

Such a space $W$ may be regarded as a rational point of a Grassmann manifold. The set of isomorphism classes of $m_{n}$ containing $m_{n-1}$ corresponds to the disjoint union of subsets of rational points of Grassmann manifolds modulo the action of D.G.A.automorphisms of $m_{D}$ (see Theorem 2.1). We can show that any minimal algebra $m$ with $H^{*}(m) \cong A^{*}$ is obtained in this way. For example if $A^{*}=H^{*}\left(S^{3} \vee\right.$ $\left.S^{5} \vee S^{16} ; Q\right)$, then $\mathcal{M}_{A^{*}}$ corresponds bijectively to $P^{3}(Q) / Q^{*} \amalg\{*\}$, where $P^{3}(Q)$ is the rational projective space of dimension 3 and the point $\{*\}$ corresponds to the formal space (see Section 3 (2)).

Throughout this paper we assume that G.A. $A^{*}$ satisfies that $A^{0}=Q, A^{1}=0$ and $\operatorname{dim}_{Q} A^{i}<\infty$ for any positive integer $i$.

\section{Inductive construction of minimal models}

In this section we construct inductively minimal algebras $m_{n}$ and G.A. maps $\sigma_{n}: H^{*}\left(m_{n}\right)(n+1) \rightarrow A^{*}$ such that $\sigma_{n}{ }^{i}$ is isomorphic for $i \leqslant n$ and monomorphic for $i=n+1$.

Suppose that we constructed a minimal algebra $m_{n-1}$ satisfying the following conditions.

$(\mathbf{1})_{\mathbf{n}-\mathbf{1}} m_{n-1}$ is generated by elements of degree $\leqslant n-1$.

$(\mathbf{2})_{\mathbf{n}-\mathbf{1}}$ There is a G.A.-map

$$
\sigma_{n-1}:\left(H^{*}\left(m_{n-1}\right)(n)\right)^{*} \rightarrow A^{*}
$$

where $\sigma_{n-1}{ }^{i}$ is isomorphic for $i \leqslant n-1$ and monomorphic for $i=n$.

Let $m_{D}$ be the minimal algebra obtained by adding generators to $m_{n-1}$ whose differentials form a basis for the kernel of $\sigma_{n-1}{ }^{n+1} \mid\left(H\left(m_{n-1}\right)(n)\right)^{n+1}$ and $\sigma_{D}$ : $\left(H\left(m_{D}\right)(n)\right)^{*} \rightarrow A^{*}$ be the induced map. We set

$$
\begin{gathered}
\operatorname{dim}_{Q} A^{n+1}=u, \quad \operatorname{dim}_{Q} A^{n+1} /(A(n))^{n+1}=s \\
\operatorname{dim}_{Q} H^{n+1}\left(m_{D}\right)=v
\end{gathered}
$$

and

$$
\operatorname{dim}_{Q} \frac{H^{n+1}\left(m_{D}\right)}{\left(H^{*}\left(m_{D}\right)(n)\right)^{n+1}}=t .
$$


Then we have

$$
u-s=v-t
$$

Let $l$ be an integer satisfying

$$
\max (0, t-s) \leqslant l \leqslant t
$$

and $W$ be a $l$-dimensional subspace of $H^{n+1}\left(m_{D}\right)$ such that

$$
W \cap\left(H^{*}\left(m_{D}\right)(n)\right)^{n+1}=\{0\} .
$$

Let $m^{W}$ be the minimal algebra obtained by adding $l$ generators whose differentials span $W$. Note that $H\left(m^{W}\right)(n)=H\left(m_{D}\right)(n)$, hence we have a G.A.map $\sigma_{D}$ : $\left(H\left(m^{W}\right)(n)\right)^{*} \rightarrow A^{*}$ and

$$
H^{n+1}\left(m^{W}\right) \oplus W=H^{n+1}\left(m_{D}\right)
$$

so that

$$
\operatorname{dim}_{Q} \frac{H^{n+1}\left(m^{W}\right)}{\left(H\left(m^{W}\right)(n)\right)^{n+1}}=t-l \leqslant s=\operatorname{dim}_{Q} \frac{A^{n+1}}{(A(n))^{n+1}} .
$$

Let $m^{W}$ be a minimal algebra obtained by adding to $m^{W}$ the cokernel of $\sigma_{D}{ }^{n}$ : $\left(H\left(m^{W}\right)(n)\right)^{n} \rightarrow A^{n}$. Then we have a G.A. map

$$
\sigma_{n}:\left(H\left(m_{n}^{W}\right)(n)\right)^{*} \rightarrow A^{*}
$$

such that $\sigma_{n}{ }^{i}$ is isomorphic for $i \leqslant n$. For a linear monomorphism

$$
\psi: H^{n+1}\left(m^{W}\right) /\left(H\left(m^{W}\right)(n)\right)^{n+1} \rightarrow A^{n+1} /(A(n))^{n+1},
$$

if the map $\sigma_{n} \oplus \psi$ can be extend to a G.A. map

$$
\sigma_{n}^{W}:\left(H\left(m^{W}{ }_{n}\right)(n+1)\right)^{*} \rightarrow A^{*},
$$

then the pair $\left(m^{W}{ }_{n}, \sigma^{W}{ }_{n}\right)$ satisfies the condition $(1)_{n}$ and $(2)_{n}$. Remark that if we take $W$ so that $\operatorname{dim}_{Q} W=t$ we can always construct a G.A. map (2.4).

Let $m_{n}$ be a minimal algebra containg $m_{n-1}$ (hence $m_{D}$ ) satisfying $(1)_{n}$ and $(2)_{n}$. Then $m_{n}$ is constructed from $m_{D}$ by taking $W$ as the kernel of $i^{*}: H^{*}\left(m_{D}\right) \rightarrow$ $H^{*}\left(m_{n}\right)$, where $i$ is the inclusion.

By Plücker embedding Grassmann manifold is a projective variety defined over $Q$. Then the $Q$-subspace $W$ corresponds to a rational point of the variety. Let $\operatorname{Gr}(v, l)(Q)$ be the set of rational points of the Grassmann manifold of $l$-dimensional $Q$-subspaces in a $v$-dimensional space $H^{n+1}\left(m_{D}\right)$. Set

$$
M_{l}=\{W \in G r(v, l)(Q) \mid W \text { satisfies }(2.3)\}
$$

satisfying (2.3). We take bases for $H^{n+1}\left(m^{W}\right) /\left(H^{*}\left(m^{W}\right)(n)\right)^{n+1}$ and $H^{*}\left(m^{W}\right)(n)^{n+1}$. If we write a basis for $W$ as a linear combinations of those bases, we see that $M_{l}$ is a Zariski open set of $\operatorname{Gr}(v, l)(Q)$ (Compare with Example (3) in Section 3). Set

$O_{l}=\left\{W \in M_{l} \mid\right.$ there is a G.A.map $\sigma^{W}$ satisfying (2.4) for some linear map $\left.\psi\right\}$.

Let $G$ be the group of D.G.A.automorphisms of $m_{D}$. Then $G$ acts on $H^{n+1}\left(m_{D}\right)$ and hence on $\operatorname{Gr}(v, l)(Q)$. Let $W$ be an element of $O_{l}$ and $\Phi$ be an element of $G$. 
Then it is easy to see that $\Phi$ can be extended to a D.G.A.isomorphism

$$
\Phi: m_{n}^{W} \rightarrow m^{\Phi(W)} \text {. }
$$

Hence $G$ also acts on $O_{l}$.

Conversely let $W_{1}, W_{2}$ be $l$-dimensional subspaces of $H^{n+1}\left(m_{D}\right)$ such that there is a D.G.A.isomorphism

$$
f: m^{W_{1}}{ }_{n} \rightarrow m^{W_{2}}{ }_{n} .
$$

Then $f \mid m_{D}=\Phi$ is an element of $G$ and

$$
\Phi\left(W_{1}\right)=W_{2} .
$$

Hence we have

Theorem 2.1. The set of isomorphism classes of minimal algebras $m_{n}$ containing a minimal algebra $m_{n-1}$ and satisfying $(1)_{n},(2)_{n}$ corresponds bijectively to the disjoint union of orbit spaces

$$
X_{n}=\coprod_{l=\max (t-s, 0)}^{t} O_{l} / G .
$$

Note that $X_{n}$ is not empty since $O_{t}$ is not empty.

Definition 2.2. $A$ G.A. $A^{*}$ is called k-intrinsically formal (abbreviated to k-I.F.) if for any minimal algebras $m$ with $H^{*}(m)=A^{*}$, the sub D.G.A. $m(k)$ is unique up to isomorphism.

Note that any G.A. $A^{*}$ is at least 2-I.F..

Let $A^{*}$ be $(n-1)$-I.F. and $m$ be arbitrary minimal algebra with $H^{*}(m) \cong A^{*}$. Set $m_{n-1}=m(n-1)$ and $i_{n-1}: m_{n-1} \rightarrow m$ be the inclusion. Then we can construct minimal algebras $m_{D}$ and $m^{W_{0}}{ }_{n}$ as previous way where $W_{0}$ is the kernel of the induced map

$$
i_{D}^{*}: H^{n+1}\left(m_{D}\right) \rightarrow H^{n+1}(m) .
$$

The inclusion $i_{D}$ can be extended to

$$
i_{n}: m^{W_{0}} \rightarrow m
$$

so that $m^{W_{0}}{ }_{n}$ and $i_{n}{ }^{*}$ satisfy $(1)_{n},(2)_{n}$. Hence $m$ can be constructed inductively as this way. Especially we have

Corollary 2.3. If $A^{*}$ is $(n-1)-I . F$. and $A^{j}=0$ for $j>n+1$. Then $O_{l}=M_{l}$ and $\mathcal{M}_{A^{*}}=X_{n}=\coprod_{\max (t-s, 0) \leqslant l \leqslant t} M_{l} / G$.

Suppose $A^{i}=0$ for $i \leqslant n$. Then $X_{k}$ is one point for $k<3 n+1$. Therefore $m_{3 n}$ is uniquely determined, i.e., $A^{*}$ is $3 n$-I.F.. This implies

Corollary 2.4. Any $n$-connected $k$-dimensional finite $C W$ complex is formal if $k \leqslant 3 n+1$. 
This result was noticed by Stasheff [8]. We see that Corollary 2.4 is best possible by the example $A^{*}=H^{*}\left(S^{3} \vee S^{3} \vee S^{8} ; Q\right)$.

The following examples are studied in the next section, where degree is denoted by suffix.

(1) $A^{*}=H^{*}\left(S^{3} \vee S^{7} \vee S^{22} ; Q\right)$, which is 20-I.F. and $u=s=1, v=t=3$ at $n=21$.

(2) $A^{*}=H^{*}\left(S^{3} \vee S^{5} \vee S^{16} ; Q\right)$, which is 14-I.F. and $u=s=1, v=t=4$ at $n=15$.

(3) $A^{*}=\wedge\left(x_{3}, y_{5}\right) \otimes Q\left[z_{8}\right] /\left(x y, x z^{2}, y z^{2}, z^{3}\right)$, which is 14 -I.F. and $u=1, s=0$, $v=5, t=4$ at $n=15$.

(4) $A^{*}=H^{*}\left(\left(S^{2} \vee S^{2}\right) \times S^{3} ; Q\right)$, which is 3-I.F. and $u=2, s=0, v=4, t=2$ at $n=4$.

(5) $A^{*}=H^{*}\left(\left(S^{3} \vee S^{3}\right) \times S^{5} ; Q\right)$, which is 6-I.F. and $u=2, s=0, v=4, t=2$ at $n=7$.

(6) $A^{*}=H^{*}\left(S^{3} \vee S^{5} \vee S^{10} \vee S^{16} ; Q\right)$, which is 8-I.F. and $u=s=v=t=1$ at $n=9$.

(7) $A^{*}=H^{*}\left(S^{5} \vee\left(S^{3} \times S^{10}\right)\right.$; $\left.Q\right)$, which is 8-I.F. and $u=s=v=t=1$ at $n=9$.

(8) $A^{*}=H^{*}\left(\left(S^{3} \times S^{8}\right) \sharp\left(S^{3} \times S^{8}\right)\right.$; Q), which is 6 -I.F. and $u=s=v=t=2$ at $n=7$. Here $\sharp$ is connected sum.

\section{Some examples}

(1) $A^{*}=H^{*}\left(S^{3} \vee S^{7} \vee S^{22} ; Q\right)=\wedge\left(x_{3}, y_{7}\right) \otimes Q\left[z_{22}\right] /\left(x y, x z, y z, z^{2}\right)$

Then $A^{*}$ is 20 -I.F. and by straightfoward calculation

$$
m_{20}=\left(\wedge\left(x, y, \theta_{9}, \theta_{11}, \theta_{13}, \theta_{15}^{1}, \theta_{15}^{2}, \theta_{17}^{1}, \theta_{17}^{2}, \theta_{19}^{1}, \theta_{19}^{2}\right), d\right)
$$

with the differential is as follows :

$d(x)=d(y)=0, d \theta_{9}=x y, d \theta_{11}=x \theta_{9}, d \theta_{13}=x \theta_{11}, d \theta_{15}^{1}=y \theta_{9}, d \theta_{15}^{2}=x \theta_{13}$, $d \theta_{17}^{1}=x \theta_{15}^{1}+y \theta_{11}, d \theta_{17}^{2}=x \theta_{15}^{2}, d \theta_{19}^{1}=x \theta_{17}^{1}+y \theta_{13}, d \theta_{19}^{2}=x \theta_{17}^{2}$.

Then at $n=21, u=s=1$ and $v=t=3$. In fact $m_{D}=m_{20}$ and $H^{22}\left(m_{D}\right)=$ $Q\left\{e_{1}, e_{2}, e_{3}\right\}$, where $e_{1}=\left[x \theta_{19}^{2}\right], e_{2}=\left[x \theta_{19}^{1}+y \theta_{15}^{2}\right]$ and $e_{3}=\left[y \theta_{15}^{1}\right]$. Let $W$ be a 2 dimensional subspace of $H^{22}\left(m_{D}\right)$ spanned by

$$
a_{1, i} e_{1}+a_{2, i} e_{2}+a_{3, i} e_{3} \quad(i=1,2),
$$

with

$$
\operatorname{rank}\left[\begin{array}{lll}
a_{1,1} & a_{2,1} & a_{3,1} \\
a_{1,2} & a_{2,2} & a_{3,2}
\end{array}\right]=2 .
$$


Let $f \in$ Aut $m_{D}=G$ be an element such that

$$
f(x)=\lambda x, \quad f(y)=\mu y, \quad \lambda, \mu \in Q^{*} .
$$

Then we have

$$
f\left(e_{1}\right)=\lambda^{7} \mu e_{1}, f\left(e_{2}\right)=\lambda^{4} \mu^{2} e_{2}, f\left(e_{3}\right)=\lambda \mu^{3} e_{3} .
$$

The set of $W$ forms $\operatorname{Gr}(3,2)(Q)$, the rational points of Grassmann manifold of 2-dimensional spaces in the 3-dimensional space $H^{22}(m(20))$. By the Plücker embedding $i: G r(3,2)(Q) \rightarrow P^{2}(Q)$,

$$
i(W)=\left[\left|\begin{array}{ll}
a_{1,1} & a_{2,1} \\
a_{1,2} & a_{2,2}
\end{array}\right|,\left|\begin{array}{ll}
a_{1,1} & a_{3,1} \\
a_{1,2} & a_{3,2}
\end{array}\right|,\left|\begin{array}{ll}
a_{2,1} & a_{3,1} \\
a_{2,2} & a_{3,2}
\end{array}\right|,\right.
$$

$G$ acts on $P^{2}(Q)$ by $f\left[x_{1}, x_{2}, x_{3}\right]=\left[\lambda^{11} \mu^{3} x_{1}, \lambda^{8} \mu^{4} x_{2}, \lambda^{5} \mu^{5} x_{3}\right]=\left[\rho x_{1}, x_{2}, \rho^{-1} x_{3}\right]$ with $\rho=\lambda^{3} \mu^{-1}$. Hence by Corollary 2.3 , we have

$$
\mathcal{M}_{A^{*}}=M_{2} / G \coprod M_{3} \simeq P^{2}(Q) / Q^{*} \coprod\{*\} .
$$

(2) $A^{*}=H^{*}\left(S^{3} \vee S^{5} \vee S^{16} ; Q\right)=\wedge\left(x_{3}, y_{5}\right) \otimes Q\left[z_{16}\right] /\left(x y, x z, y z, z^{2}\right)$

Then $A^{*}$ is 14 -I.F. and by straightfoward calculation

$$
m_{D}=m_{14}=\left(\wedge\left(x, y, \theta_{7}, \theta_{9}, \theta_{11}^{1}, \theta_{11}^{2}, \theta_{13}^{1}, \theta_{13}^{2}\right), d\right)
$$

with the differential is as follows:

$d(x)=d(y)=0, d \theta_{7}=x y, d \theta_{9}=x \theta_{7}, d \theta_{11}^{1}=y \theta_{7}, d \theta_{11}^{2}=x \theta_{9}, d \theta_{13}^{1}=x \theta_{11}^{2}$, $d \theta_{13}^{2}=x \theta_{11}^{1}+y \theta_{9}$.

Then at $n=15, u=s=1$ and $H^{16}\left(m_{D}\right)=Q\left\{e_{1}, e_{2}, e_{3}, e_{4}\right\}$, where $e_{1}=\left[x \theta_{13}^{1}\right]$, $e_{2}=\left[y \theta_{11}^{1}\right], e_{3}=\left[x \theta_{13}^{2}+\theta_{7} \theta_{9}\right]$ and $e_{4}=\left[y \theta_{11}^{2}+\theta_{7} \theta_{9}\right]$. Hence at $n=15, v=t=4$. Let $W$ be a 3 -dimensional subspace of $H^{16}\left(m_{D}\right)$ spanned by

$$
a_{1, i} e_{1}+a_{2, i} e_{2}+a_{3, i} e_{3}+a_{4, i} e_{4} \quad(i=1,2,3),
$$

where $\operatorname{rank}\left(a_{j, i}\right)_{1 \leqslant j \leqslant 4,1 \leqslant i \leqslant 3}=3$.

Let $f \in$ Aut $m_{D}=G$ be an element such that

$$
f(x)=\lambda x, \quad f(y)=\mu y, \quad \lambda, \mu \in Q^{*} .
$$

Then we have

$$
f\left(e_{1}\right)=\lambda^{5} \mu e_{1}, f\left(e_{2}\right)=\lambda \mu^{3} e_{2}, f\left(e_{3}\right)=\lambda^{3} \mu^{2} e_{3}, f\left(e_{4}\right)=\lambda^{3} \mu^{2} e_{4} .
$$

The set of $W$ forms $\operatorname{Gr}(4,3)(Q)$, which is isomorphic to $P^{3}(Q)$ by the Plücker embedding $i: G r(4,3)(Q) \rightarrow P^{3}(Q)$,

$$
i(W)=\left[\left|\begin{array}{lll}
\mid a_{1,1} & a_{2,1} & a_{3,1} \\
a_{1,2} & a_{2,2} & a_{3,2} \\
a_{1,3} & a_{2,3} & a_{3,3}
\end{array}\right|,\left|\begin{array}{lll}
a_{1,1} & a_{2,1} & a_{4,1} \\
a_{1,2} & a_{2,2} & a_{4,2} \\
a_{1,3} & a_{2,3} & a_{4,3}
\end{array}\right|,\left|\begin{array}{lll}
a_{1,1} & a_{3,1} & a_{4,1} \\
a_{1,2} & a_{3,2} & a_{4,2} \\
a_{1,3} & a_{3,3} & a_{4,3}
\end{array}\right|,\left|\begin{array}{lll}
a_{2,1} & a_{3,1} & a_{4,1} \\
a_{2,2} & a_{3,2} & a_{4,2} \\
a_{2,3} & a_{3,3} & a_{4,3}
\end{array}\right|\right] .
$$

Then $G$ acts on $P^{2}(Q)$ by $f\left[x_{1}, x_{2}, x_{3}, x_{4}\right]=\left[\lambda^{9} \mu^{6} x_{1}, \lambda^{9} \mu^{6} x_{2}, \lambda^{11} \mu^{5} x_{3}, \lambda^{7} \mu^{7} x_{4}\right]=$ $\left[\rho x_{1}, \rho x_{2}, \rho^{2} x_{3}, x_{4}\right]$ by putting $\rho=\lambda^{2} \mu^{-1}$. Hence by Corollary 2.3, we have

$$
\mathcal{M}_{A^{*}}=M_{3} / G \coprod M_{4} \simeq P^{3}(Q) / Q^{*} \coprod\{*\} .
$$

(3) $A^{*}=\wedge\left(x_{3}, y_{5}\right) \otimes Q\left[z_{8}\right] /\left(x y, x z^{2}, y z^{2}, z^{3}\right)$ 
Then $A^{*}$ is $14-$ I.F. and at $n=15, u=1, s=0$, and

$$
m_{D}=m_{14}=m^{\prime}{ }_{14} \otimes Q[z],
$$

where $m^{\prime}{ }_{14}$ is isomorphic to $m_{14}$ in the example $(2)$ and $d(z)=0$. Then $H^{16}\left(m_{D}\right)=$ $Q\left\{e_{1}, e_{2}, e_{3}, e_{4}, f_{1}\right\}$, where $e_{1}=\left[x \theta_{13}^{1}\right], e_{2}=\left[y \theta_{11}^{1}\right], e_{3}=\left[x \theta_{13}^{2}+\theta_{7} \theta_{9}\right], e_{4}=\left[y \theta_{11}^{2}+\right.$ $\left.\theta_{7} \theta_{9}\right]$ and $f_{1}=\left[z^{2}\right]$. Hence at $n=15, v=5, t=4$. By Corollary 2.3,

$$
\mathcal{M}_{A^{*}}=X_{15}=M_{4} / G \text {. }
$$

Let $W$ be an element of $M_{4}$ spanned by

$$
a_{1, i} e_{1}+a_{2, i} e_{2}+a_{3, i} e_{3}+a_{4, i} e_{4}+a_{5, i} f_{1} \quad(i=1,2,3,4),
$$

with

$$
\operatorname{rank}\left(a_{j, i}\right)_{1 \leqslant j \leqslant 4,1 \leqslant i \leqslant 4}=4 \quad(*) .
$$

By Plücker embedding, we see that the set of $W$ satisfying $(*)$ corresponds bijectively to $A^{4}(Q)=\left\{\left[x_{1}, x_{2}, x_{3}, x_{4}, x_{5}\right] \in P^{4}(Q) \mid x_{1} \neq 0\right\}$.

Let $f \in$ Aut $m_{D}=G$ be an element such that

$$
f(x)=\lambda x, \quad f(y)=\mu y, \quad f(z)=\kappa z, \quad \lambda, \mu, \kappa \in Q^{*} .
$$

Then we have

$$
\begin{gathered}
f^{*}\left(e_{1}\right)=\lambda^{5} \mu e_{1}, f^{*}\left(e_{2}\right)=\lambda \mu^{3} e_{2}, f^{*}\left(e_{3}\right)=\lambda^{3} \mu^{2} e_{3}, \\
f^{*}\left(e_{4}\right)=\lambda^{3} \mu^{2} e_{4}, f^{*}\left(f_{1}\right)=\kappa^{2} f_{1} .
\end{gathered}
$$

Hence $G$ acts on $P^{4}(Q)$ by

$$
f \cdot\left[x_{1}, x_{2}, x_{3}, x_{4}, x_{5}\right]=\left[\lambda^{12} \mu^{8} x_{1}, \lambda^{11} \mu^{5} \kappa^{2} x_{2}, \lambda^{9} \mu^{6} \kappa^{2} x_{3}, \lambda^{9} \mu^{6} \kappa^{2} x_{4}, \lambda^{7} \mu^{7} \kappa^{2} x_{5}\right] .
$$

Hence $G$ acts on $A^{4}(Q)$ by

$$
f \cdot\left(y_{1}, y_{2}, y_{3}, y_{4}\right)=\left(\lambda^{-1} \mu^{-3} \kappa^{2} y_{1}, \lambda^{-3} \mu^{-2} \kappa^{2} y_{2}, \lambda^{-3} \mu^{-2} \kappa^{2} y_{3}, \lambda^{-5} \mu^{-1} \kappa^{2} y_{4}\right),
$$

where $y_{i}=x_{i+1} / x_{1}$ for $i=1, . ., 4$. Then setting $\alpha=\lambda^{-7} \kappa^{2}$ and $\beta=\lambda^{2} \mu^{-1}, G$ acts on $A^{4}(Q)$ by

$$
f \cdot\left(y_{1}, y_{2}, y_{3}, y_{4}\right)=\left(\alpha \beta^{3} y_{1}, \alpha \beta^{2} y_{2}, \alpha \beta^{2} y_{3}, \alpha \beta y_{4}\right) .
$$

Since $\alpha$ and $\beta$ take any non-zero rational numbers independently, we have

$$
\mathcal{M}_{A^{*}} \simeq A^{4}(Q) /\left(Q^{*} \times Q^{*}\right) \simeq P^{3}(Q) / Q^{*} \coprod\{*\},
$$

where $Q^{*}$ acts on $P^{3}(Q)$ by

$$
\beta \cdot\left[z_{1}, z_{2}, z_{3}, z_{4}\right]=\left[\beta^{2} z_{1}, \beta z_{2}, \beta z_{3}, z_{4}\right]
$$

and the point $\{*\}$ corresponds $(0,0,0,0)$ in $A^{4}(Q)$, which corresponds a formal model. Thus $\mathcal{M}_{A^{*}}$ is the same set as that of Example (2).

(4) $A^{*}=H^{*}\left(\left(S^{2} \vee S^{2}\right) \times S^{3} ; Q\right)=Q\left[x_{2}, y_{2}\right] \otimes \Lambda\left(z_{3}\right) /(x y)$.

This example was studied by Halperin and Stasheff, see example 6.5 in [2]. It is 3 -I.F. and at $n=4, s=0$ and $t=2$. In fact

$$
m_{D}=m_{3}=\left(\wedge\left(x, y, \theta_{3}^{1}, \theta_{3}^{2}, \theta_{3}^{3}, z_{3}\right), d\right)
$$


with $d(x)=d(y)=d(z)=0, d \theta_{3}^{1}=x^{2}, d \theta_{3}^{2}=x y, d \theta_{3}^{3}=y^{2}$ and $H^{5}\left(m_{3}\right)=$ $Q\left\{e_{1}, e_{2}, f_{1}, f_{2}\right\}$, where $e_{1}=\left[y \theta_{3}^{1}-x \theta_{3}^{2}\right], e_{2}=\left[y \theta_{3}^{2}-x \theta_{3}^{3}\right], f_{1}=[x z]$ and $f_{2}=[y z]$. Then by Collorary 2.3,

$$
\mathcal{M}_{A^{*}}=X_{4}=M_{2} / G
$$

Let $W$ in $M_{2}$ be spanned by

$$
a_{1, i} e_{1}+a_{2, i} e_{2}+a_{3, i} f_{1}+a_{4, i} f_{2} \quad(i=1,2),
$$

where

$$
\operatorname{rank}\left(a_{j, i}\right)_{1 \leqslant j \leqslant 2,1 \leqslant i \leqslant 2}=2 .
$$

By Plücker embedding, the set of $W$ forms

$$
\begin{gathered}
\left\{\left[x_{1}, x_{2}, x_{3}, x_{4}, x_{5}, x_{6}\right] \in P^{5}(Q) \mid x_{1} x_{6}-x_{2} x_{5}+x_{3} x_{4}=0, x_{1} \neq 0\right\} \\
\simeq\left\{\left(X_{1}, X_{2}, X_{3}, X_{4}, X_{5}\right) \in A^{5}(Q) \mid X_{5}-X_{2} X_{5}+X_{3} X_{4}=0\right\} \\
\simeq\left\{\left(X_{1}, X_{2}, X_{3}, X_{4}\right) \in A^{4}(Q)\right\}
\end{gathered}
$$

where $X_{i}=x_{i+1} / x_{1}(i=1, . ., 5)$.

Let $f \in$ Aut $m_{D}=G$ be an element such that

$$
\begin{gathered}
f(x)=x, \quad f(y)=y, \quad f(z)=\mu z \quad \mu \in Q^{*} \\
f\left(\theta_{3}^{i}\right)=\theta_{3}^{i}+\lambda_{i} z, \quad \lambda_{i} \in Q, \quad i=1,2,3 .
\end{gathered}
$$

Then we have

$$
\begin{gathered}
f^{*}\left(e_{1}\right)=e_{1}-\lambda_{2} f_{1}+\lambda_{1} f_{2}, f^{*}\left(e_{2}\right)=e_{2}-\lambda_{3} f_{1}+\lambda_{2} f_{2}, \\
f^{*}\left(f_{1}\right)=\mu f_{1}, f^{*}\left(f_{2}\right)=\mu f_{2},
\end{gathered}
$$

and $f^{*}$ induces a map $A_{f}$ defined by

$$
A_{f}\left(\left[x_{1}, . ., x_{6}\right]\right)=\left[x_{1}, . ., x_{6}\right]\left[\begin{array}{cccccc}
1 & -\lambda_{3} & \lambda_{2} & \lambda_{2} & -\lambda_{1} & \lambda_{1} \lambda_{3}-\lambda_{2}^{2} \\
0 & \mu & 0 & 0 & 0 & -\lambda_{1} \mu \\
0 & 0 & \mu & 0 & 0 & -\lambda_{2} \mu \\
0 & 0 & 0 & \mu & 0 & -\lambda_{2} \mu \\
0 & 0 & 0 & 0 & \mu & -\lambda_{3} \mu \\
0 & 0 & 0 & 0 & 0 & \mu^{2}
\end{array}\right],
$$

hence $f^{*}$ induces a map $\tilde{A}_{f}$ from $A^{4}(Q)$ to itself defined by

$$
\tilde{A}_{f}\left[\begin{array}{c}
X_{1} \\
X_{2} \\
X_{3} \\
X_{4}
\end{array}\right]=\left[\begin{array}{llll}
\mu & & & \\
& \mu & & \\
& & \mu & \\
& & & \mu
\end{array}\right]\left[\begin{array}{c}
X_{1} \\
X_{2} \\
X_{3} \\
X_{4}
\end{array}\right]+\left[\begin{array}{c}
-\lambda_{3} \\
\lambda_{2} \\
\lambda_{2} \\
-\lambda_{1}
\end{array}\right] .
$$

From this we see by varing $\lambda_{i} \in Q(i=1,2,3)$ and $\mu \in Q^{*}$,

$$
\tilde{A}_{f}\left[\begin{array}{l}
0 \\
0 \\
0 \\
0
\end{array}\right] \cup \tilde{A}_{f}\left[\begin{array}{l}
0 \\
1 \\
0 \\
0
\end{array}\right]=A^{4}(Q) \text {. }
$$


Hence $\mathcal{M}_{A^{*}}$ is at most two points.

Conversely, any element $g \in A$ ut $m_{D}$ has the following form: $g(x)=a_{1} x+a_{2} y$, $g(y)=b_{1} x+b_{2} y$ and $g(z)=\mu z$ with

$$
a_{1}, a_{2}, b_{1}, b_{2} \in Q, D=\left|\begin{array}{ll}
a_{1} & a_{2} \\
b_{1} & b_{2}
\end{array}\right| \neq 0, \mu \in Q^{*}
$$

and then

$g\left(\theta_{1}\right)=a_{1}^{2} \theta_{1}+2 a_{1} a_{2} \theta_{2}+a_{2}^{2} \theta_{3}+\lambda_{1} z$

$g\left(\theta_{2}\right)=a_{1} b_{1} \theta_{1}+\left(a_{1} b_{2}+a_{2} b_{1}\right) \theta_{2}+a_{2} b_{2} \theta_{3}+\lambda_{2} z$,

$g\left(\theta_{3}\right)=b_{1}^{2} \theta_{1}+2 b_{1} b_{2} \theta_{2}+b_{2}^{2} \theta_{3}+\lambda_{3} z$

for some $\lambda_{i} \in Q$. By straightfoward calculations we see that $W_{1}=\left\{e_{1}, e_{2}\right\}$, which corresponds to $(0,0,0,0)$ in $A^{4}(Q)$, can not be mapped to $W_{2}=\left\{e_{1}, e_{2}+f_{2}\right\}$ corresponding to $(0,1,0,0)$ in $A^{4}(Q)$ by Aut $m_{D}$. In fact,

$$
\tilde{A}_{g}\left[\begin{array}{l}
0 \\
0 \\
0 \\
0
\end{array}\right]=\frac{1}{D^{2}} \cdot\left[\begin{array}{c}
-b_{1}^{2} \lambda_{1}+2 a_{1} b_{1} \lambda_{2}-a_{1}^{2} \lambda_{3} \\
-b_{1} b_{2} \lambda_{1}+\left(a_{1} b_{2}+a_{2} b_{1}\right) \lambda_{2}-a_{1} a_{2} \lambda_{3} \\
-b_{1} b_{2} \lambda_{1}+\left(a_{1} b_{2}+a_{2} b_{1}\right) \lambda_{2}-a_{1} a_{2} \lambda_{3} \\
-b_{2}^{2} \lambda_{1}+2 a_{2} b_{2} \lambda_{2}-a_{2}^{2} \lambda_{3}
\end{array}\right]=\left[\begin{array}{c}
* \\
\alpha \\
\alpha \\
*
\end{array}\right] \neq\left[\begin{array}{l}
0 \\
1 \\
0 \\
0
\end{array}\right] .
$$

Thus we see that $\mathcal{M}_{A^{*}}$ is just two points.

(5) $A^{*}=H^{*}\left(\left(S^{3} \vee S^{3}\right) \times S^{5} ; Q\right)=\Lambda\left(x_{3}, y_{3}, z_{5}\right) /(x y)$.

This example was considered by Schlessinger and Stasheff, see section 8 in [7]. It is 6-I.F. and

$$
m_{D}=m_{6}=\left(\wedge\left(x_{3}, y_{3}, \theta_{5}, z_{5}\right), d\right)
$$

with $d(x)=d(y)=d(z)=0$ and $d \theta_{5}=x y$. Then $H^{8}\left(m_{D}\right)=Q\left\{e_{1}, e_{2}, f_{1}, f_{2}\right\}$, where $e_{1}=\left[x \theta_{5}\right], e_{2}=\left[y \theta_{5}\right], f_{1}=[x z]$ and $f_{2}=[y z]$. Hence at $n=7, s=0$ and $t=2$. By Corollary 2.3,

$$
\mathcal{M}_{A^{*}}=X_{7}=M_{2} / G
$$

Let $W$ in $M_{2}$ be spanned by

$$
a_{1, i} e_{1}+a_{2, i} e_{2}+a_{3, i} f_{1}+a_{4, i} f_{2} \quad(i=1,2),
$$

where $\operatorname{rank}\left(a_{j, i}\right)_{1 \leqslant j \leqslant 2,1 \leqslant i \leqslant 2}=2$.

Let $f \in$ Aut $m_{D}=G$ be an element such that $f(x)=a_{1} x+a_{2} y, f(y)=b_{1} x+b_{2} y$, $f\left(\theta_{5}\right)=D \theta_{5}+\lambda z$ and $f(z)=\mu z$, where

$$
D=\left|\begin{array}{ll}
a_{1} & a_{2} \\
b_{1} & b_{2}
\end{array}\right| \neq 0, \lambda \in Q, \mu \in Q^{*} .
$$

Then

$$
\begin{gathered}
f^{*}\left(e_{1}\right)=a_{1} D e_{1}+a_{2} D e_{2}+a_{1} \lambda f_{1}+a_{2} \lambda f_{2}, \\
f^{*}\left(e_{2}\right)=b_{1} D e_{1}+b_{2} D e_{2}+b_{1} \lambda f_{1}+b_{2} \lambda f_{2}, \\
f^{*}\left(f_{1}\right)=a_{1} \mu f_{1}+a_{2} \mu f_{2}, f^{*}\left(f_{2}\right)=b_{1} \mu f_{1}+b_{2} \mu f_{2} .
\end{gathered}
$$


By Plücker embedding the set of $W$ forms

$$
\begin{gathered}
\left\{\left[x_{1}, x_{2}, x_{3}, x_{4}, x_{5}, x_{6}\right] \in P^{5}(Q) \mid x_{1} x_{6}-x_{2} x_{5}+x_{3} x_{4}=0, x_{1} \neq 0\right\} \\
\simeq\left\{\left(X_{1}, X_{2}, X_{3}, X_{4}\right) \in A^{4}(Q)\right\}
\end{gathered}
$$

where $X_{i}=x_{i+1} / x_{1}(i=1, . ., 4)$. Then $G$ acts on $A^{4}(Q)$ as follows:

$$
\tilde{A}_{f}\left[\begin{array}{l}
X_{1} \\
X_{2} \\
X_{3} \\
X_{4}
\end{array}\right]=\frac{\mu}{D^{2}}\left[\begin{array}{cccc}
a_{1}^{2} & a_{1} b_{1} & a_{1} b_{1} & b_{1}^{2} \\
a_{1} a_{2} & a_{1} b_{2} & a_{2} b_{1} & b_{1} b_{2} \\
a_{1} a_{2} & a_{2} b_{1} & a_{1} b_{2} & b_{1} b_{2} \\
a_{2}^{2} & a_{2} b_{2} & a_{2} b_{2} & b_{2}^{2}
\end{array}\right]\left[\begin{array}{l}
X_{1} \\
X_{2} \\
X_{3} \\
X_{4}
\end{array}\right]+\frac{\lambda}{D}\left[\begin{array}{c}
0 \\
1 \\
-1 \\
0
\end{array}\right] .
$$

First we show that any point $\left(x_{1}, x_{2}, x_{3}, x_{4}\right)$ of $A^{4}(Q)$ lies in the union of the orbit of $(1,0,0, r)$ for some $r \in Q$ and that of $(0,0,0,0)$ by decomposing $A^{4}(Q)$ into the following pieces $(\mathrm{a}) \sim(\mathrm{f})$.

(a) If $4 x_{1} x_{4} \neq\left(x_{2}+x_{3}\right)^{2}$ and $x_{1} \neq 0$, set $a_{1}=0, a_{2}=-1, b_{1}=1, b_{2}=-\frac{x_{2}+x_{3}}{2 x_{1}}$, $\mu=\frac{\left(x_{2}+x_{3}\right)^{2}-4 x_{1} x_{4}}{4 x_{1}}, r=\frac{4 x_{1}^{2}}{\left(x_{2}+x_{3}\right)^{2}-4 x_{1} x_{4}}$ and $\lambda=\frac{1}{2}\left(x_{2}-x_{3}\right)$. Then we have

$$
\tilde{A}_{f}\left[\begin{array}{l}
1 \\
0 \\
0 \\
r
\end{array}\right]=\left[\begin{array}{l}
x_{1} \\
x_{2} \\
x_{3} \\
x_{4}
\end{array}\right] .
$$

(b) If $4 x_{1} x_{4} \neq\left(x_{2}+x_{3}\right)^{2}$ and $x_{4} \neq 0$, set $a_{1}=1, a_{2}=0, b_{1}=-\frac{x_{2}+x_{3}}{2 x_{4}}, b_{2}=1$, $\mu=\frac{\left(x_{2}+x_{3}\right)^{2}-4 x_{1} x_{4}}{4 x_{4}}, r=\frac{4 x_{4}^{2}}{\left(x_{2}+x_{3}\right)^{2}-4 x_{1} x_{4}}$ and $\lambda=\frac{1}{2}\left(x_{2}-x_{3}\right)$. Then we have (3.1).

(c) If $4 x_{1} x_{4} \neq\left(x_{2}+x_{3}\right)^{2}$ and $x_{1}=x_{4}=0$, set $a_{1}=b_{1}=1, a_{2}=-\frac{1}{2}, b_{2}=\frac{1}{2}$ $\mu=-\frac{x_{2}+x_{3}}{2}, r=-2$ and $\lambda=\frac{1}{2}\left(x_{2}-x_{3}\right)$. Then we have (3.1).

(d) If $4 x_{1} x_{4}=\left(x_{2}+x_{3}\right)^{2}$ and $x_{1} \neq 0$, set $a_{1}=x_{1}, a_{2}=-\frac{x_{2}+x_{3}}{2}, b_{1}=0, b_{2}=\frac{1}{x_{1}}$ $\mu=-\frac{1}{x_{1}}, r=0$ and $\lambda=\frac{1}{2}\left(x_{2}-x_{3}\right)$. Then we have (3.1).

(e) If $4 x_{1} x_{4}=\left(x_{2}+x_{3}\right)^{2}$ and $x_{4} \neq 0$, set $a_{1}=-\frac{x_{2}+x_{3}}{2}, a_{1}=x_{4}, b_{1}=-\frac{1}{x_{1}}, b_{2}=$ $0, \mu=-\frac{1}{x_{4}}, r=0$ and $\lambda=\frac{1}{2}\left(x_{2}-x_{3}\right)$. Then we have (3.1).

(f) If $x_{1}=x_{4}=0, x_{2}+x_{3}=0$, set $a_{1}=1, a_{2}=0, b_{1}=0, b_{2}=1, \mu=1$ and $\lambda=x_{2}$. Then we have

$$
\tilde{A}_{f}\left[\begin{array}{l}
0 \\
0 \\
0 \\
0
\end{array}\right]=\left[\begin{array}{c}
0 \\
x_{2} \\
x_{3} \\
0
\end{array}\right]
$$

Thus we have a surjection

$$
p: Q \coprod\{*\} \rightarrow \mathcal{M}_{A^{*}} \simeq A^{4}(Q) / G
$$

defined by $p(*)=$ the class of $(0,0,0,0)$ and $p(r)=$ the class of $(1,0,0, r)$. 
If $p\left(r_{1}\right)=p\left(r_{2}\right)$ then there is an element $f \in G$ such that

$$
\tilde{A}_{f}\left[\begin{array}{l}
1 \\
0 \\
0 \\
r_{1}
\end{array}\right]=\left[\begin{array}{c}
1 \\
0 \\
0 \\
r_{2}
\end{array}\right] .
$$

By straightfoward calculations we have $r_{1} r_{2} \in Q^{* 2}$ if $r_{1} r_{2} \neq 0$. Thus we have

$$
\mathcal{M}_{A^{*}} \simeq Q^{*} / Q^{* 2} \coprod\{0\} \coprod\{*\},
$$

where $\{0\}$ corresponds to $(1,0,0,0)$ and $\{*\}$ corresponds to the formal model. After tensoring with $\bar{Q}$ the set of isomorphism classes consists of three points.

(6) $A^{*}=H^{*}\left(S^{3} \vee S^{5} \vee S^{10} \vee S^{16} ; Q\right)=\wedge\left(x_{3}, y_{5}\right) \otimes$ $Q\left[v_{10}, z_{16}\right] /\left(x y, x v, x z, y v, y z, v^{2}, v z, z^{2}\right)$.

Then $m_{D}=m_{8}=\left(\Lambda\left(x, y, \theta_{7}\right), d\right)$ with $d\left(\theta_{7}\right)=x y$. Since $H^{10}\left(m_{8}\right)=Q\left\{x \theta_{7}\right\}$, $s=t=1$ at $n=9$. Then since the condition $(2)_{9}$ is satisfied

$$
X_{9}=O_{0} \coprod O_{1}=M_{0} \coprod M_{1} \simeq\left\{p_{0}, p_{1}\right\},
$$

where the corresponding model for $p_{0}$ is

$$
m_{9}^{(0)}=\left(\Lambda\left(x, y, \theta_{7}\right), d\right)
$$

with $d\left(\theta_{7}\right)=x y$ and the corresponding model for $p_{1}$ is

$$
m_{9}^{(1)}=\left(\Lambda\left(x, y, \theta_{7}, \theta_{9}\right), d\right)
$$

with $d\left(\theta_{9}\right)=x \theta_{7}$.

Next consider $X_{15}$ over each point. The model containing $m^{(0)}{ }_{9}$ is

$$
m_{D}=m_{14}=\left(\wedge\left(x, y, \theta_{7}, \theta_{11}\right), d\right)
$$

with $d\left(\theta_{11}\right)=y \theta_{7}$. Since $H^{16}\left(m_{D}\right)=Q\left\{y \theta_{11}\right\}, s=t=1$ at $n=15$. Hence $X_{15}$ consists of two points.

The model containing $m^{(1)}{ }_{9}$ is

$$
m_{D}=m_{14}=\left(\Lambda\left(x, y, \theta_{7}, \theta_{9}, u_{10}, \theta_{11}^{1}, \theta_{11}^{2}, \theta_{13}^{1}, \theta_{13}^{2}\right), d\right)=(Q[u] \otimes m, d)
$$

where $d\left(u_{10}\right)=0$ for a basis $u_{10}$ of $\operatorname{Coker}\left(\sigma_{9}^{\left\{x \theta_{7}\right\}}\right)^{10}$ and $m$ is the model $(*)$ in Example (2). Then $H^{16}\left(m_{D}\right)=Q\left\{e_{1}, e_{2}, e_{3}, e_{4}\right\}$ is same as that of the above Example (2). Hence we have in this case

$$
X_{15} \simeq \mathcal{M}_{H^{*}\left(S^{3} \vee S^{5} \vee S^{16}\right)} .
$$

Since $A^{>16}=0, \mathcal{M}_{A^{*}}$ is the disjoint union of two points and $P^{3}(Q) / Q^{*} \amalg\{*\}$. See Fig 1.

(7) $A^{*}=H^{*}\left(S^{5} \vee\left(S^{3} \times S^{10}\right) ; Q\right)=\Lambda\left(x_{3}, y_{5}\right) \otimes Q\left[z_{10}\right] /\left(x y, x z, z^{2}\right)$.

Then $m_{D}=m_{8}=\left(\Lambda\left(x, y, \theta_{7}\right), d\right)$ with $d\left(\theta_{7}\right)=x y$. Since $H^{10}\left(m_{8}\right)=Q\left\{x \theta_{7}\right\}$, $W=0$ or $W=Q\left\{x \theta_{7}\right\}$ at $n=9$. If $W=\{0\},\left(\sigma^{W}\right)^{13}: H^{3}\left(m^{W}\right) \cdot H^{10}\left(m^{W}\right)=$ 
$0 \rightarrow A^{3} \cdot A^{10} \neq 0$ can not be a G.A.map. Hence the condition $(2)_{9}$ is not satisfied. Hence $W$ must be $Q\left\{x \theta_{7}\right\}$.

Next consider $X_{12}$. Then

$$
m_{D}=m_{12}=\left(\Lambda\left(x, y, \theta_{7}, \theta_{9}, u_{10}, \theta^{1}{ }_{11}, \theta^{2}{ }_{11}\right), d\right)
$$

with $d\left(\theta_{7}\right)=x y, d\left(\theta_{9}\right)=x \theta_{7}, d\left(\theta^{1}{ }_{11}\right)=y \theta_{7}, d\left(\theta^{2}{ }_{11}\right)=x \theta_{9}$. Since $H^{13}\left(m_{D}\right)=\left(H^{+}\left(m_{D}\right)(12)\right)^{13}$ and $A^{>13}=0, \mathcal{M}_{A^{*}}$ is an one point.

(8) $A^{*}=H^{*}\left(\left(S^{3} \times S^{8}\right) \sharp\left(S^{3} \times S^{8}\right) ; Q\right)=\Lambda\left(x_{3}, y_{3}\right) \otimes Q\left[u_{8}, w_{8}\right] /(x y, x u, x w+$ $\left.y u, y w, u^{2}, u w, w^{2}\right)$.

It is 6-intrinsically formal Poincaré algebra of formal dimension 11 such that $m_{6}=\left(\Lambda\left(x, y, \theta_{5}\right), d\right)$ with $d(x)=d(y)=0$ and $d\left(\theta_{5}\right)=x y$. There is a map $\sigma_{6}:\left(H^{*}\left(m_{6}\right)(7)\right)^{*} \rightarrow A^{*}$ given by $\sigma_{6}(x)=x, \sigma_{6}(y)=y$ and sending other elements to zero. Since $u=s=v=t=2$ at $n=7$, we have $0 \leqslant l \leqslant 2$. Consider the each cases of $l=0,1,2$ at $n=7$ in the followings.

Case of $l=0$.

Since $W=0, H^{8}\left(m^{W}\right)=H^{8}\left(m_{6}\right)=Q\left\{\left[x \theta_{5}\right],\left[y \theta_{5}\right]\right\}$. Put $\sigma^{W}(x)=x, \sigma^{W}(y)=$ $y, \sigma^{W}\left(\left[x \theta_{5}\right]\right)=u$ and $\sigma^{W}\left(\left[y \theta_{5}\right]\right)=w$. Then the condition $(1)_{7}$ and $(2)_{7}$ are satisfied. Since $\sigma^{W}: H^{*}\left(m^{W}\right) \rightarrow A^{*}$ is isomorphic, this one point set $M_{0}=O_{0}$, corresponding the elliptic model $\left(\Lambda\left(x, y, \theta_{5}\right), d\right)$, is a component of $\mathcal{M}_{A^{*}}$.

Case of $l=1$.

For $H^{8}\left(m_{6}\right)=Q\left\{e_{1}=\left[x \theta_{5}\right], e_{2}=\left[y \theta_{5}\right]\right\}, W$ is spanned by $a e_{1}+b e_{2}$ for $[a, b] \in P^{1}(Q)=M_{1}$. Then $m^{W}{ }_{8}=\left(\Lambda\left(x, y, \theta_{5}, \theta_{7}, u_{8}\right), d\right)$ where $d\left(\theta_{7}\right)=a e_{1}+b e_{2}$ and $d\left(u_{8}\right)=0$. But $\left(\sigma_{8}^{W_{8}}\right)^{11}: H^{3}\left(m^{W}\right) \cdot H^{8}\left(m^{W}\right) \rightarrow A^{3} \cdot A^{8}$ can not be a G.A.map since $x \cdot\left(b x \theta_{5}+a y \theta_{5}\right)=d\left(y \theta_{7}\right)$ and $y \cdot\left(b x \theta_{5}+a y \theta_{5}\right)=d\left(x \theta_{7}\right)$. Hence the condition $(2)_{7}$ is not satisfied.

Case of $l=2$.

Since $W=Q\left\{x \theta_{5}, y \theta_{5}\right\}$,

$$
m^{W}=\left(\Lambda\left(x, y, \theta_{5}, \theta^{1}{ }_{7}, \theta^{2}{ }_{7}\right), d\right)
$$

where $d\left(\theta^{1}{ }_{7}\right)=x \theta_{5}$ and $d\left(\theta^{2}{ }_{7}\right)=y \theta_{5}$ and

$$
m^{W}{ }_{8}=\left(\Lambda\left(x, y, \theta_{5}, \theta_{7}^{1}, \theta^{2}{ }_{7}, u_{8}^{1}, u_{8}^{2}\right), d\right)
$$

where $d u^{i}{ }_{8}=0(i=1,2)$. Since $t=0$ at $8 \leqslant n \leqslant 11$ and $A^{>11}=0$, it is one point corresponding to the formal model.

Thus $\mathcal{M}_{A^{*}}$ is two points. See Fig 2 . 
In the following figures, numbers mean degrees.

Fig 1

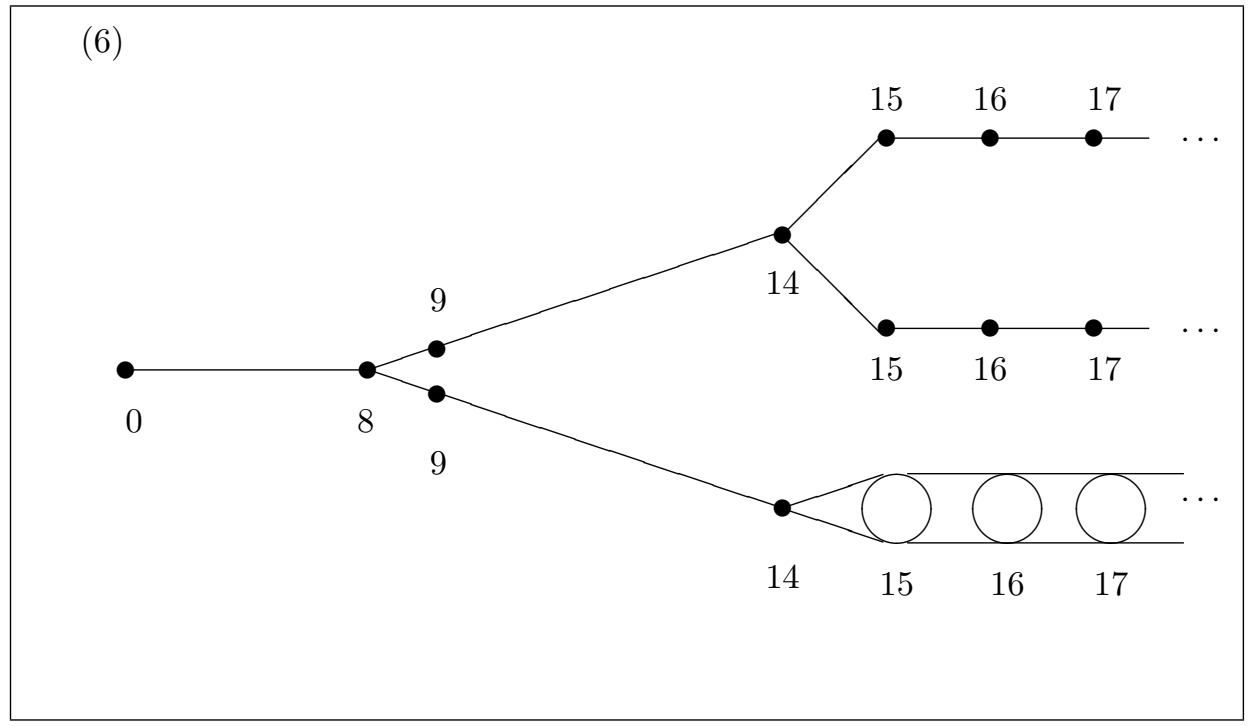

The set $P^{3}(Q) / Q^{*} \coprod\{*\}$ is indicated by one circle.

Fig 2

(8)

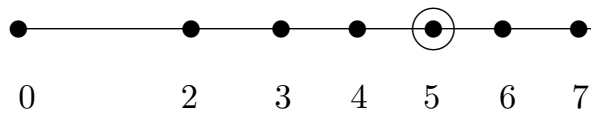

Here $\odot$ implies that there exists an elliptic minimal model generated by elements of degree $\leqslant 5$ satisfying $H^{*}(m) \cong A^{*}$. 


\section{References}

[1] Felix, Y., [1979] Classification homotopique des espaces rationals de cohomologie donnee, Bull. Soc. Math. of Belgique, 31, 75-86.

[2] Halperin,S. and Stasheff,J., [1979] Obstructions to homotopy equivalences, Advance in Math., 32, 233-279.

[3] Lupton, G., [1991] Algebras realized by $n$ rational homotopy types, Proceedings of the A.M.S., 113, 1179-1184.

[4] Neisendorfer, J. and Miller, T., [1978] Formal and coformal spaces, Illinois J.of Math. 22, 565-580.

[5] Nishimoto, T., Shiga, H. and Yamaguchi, T., [2003] Elliptic rational spaces whose cohomologies and homotopies are isomorphic, Topology, 42, 1397-1401.

[6] Nishimoto, T., Shiga, H. and Yamaguchi, T., Rationally elliptic spaces with isomorphic cohomology algebras, to appear in JPAA.

[7] Schlessinger, M. and Stasheff, J., (1991) Deformation theory and rational homotopy type, preprint.

[8] Stasheff,J., [1983] Rational Poincaré duality spaces, Illinois J. of Math., 27, 104-109.

[9] Sullivan, D., [1978] Infinitesimal computations in topology, Publ. Math. of I.H.E.S. 47, 269-331.

[10] Shiga, H. and Yagita, N., [1982] Graded algebras having a unique rational homotopy type, Proceedings of the A.M.S., 85, 623-632.

This article may be accessed via WWW at http://www.rmi.acnet.ge/hha/

or by anonymous ftp at

ftp://ftp.rmi.acnet.ge/pub/hha/volumes/2003/n1a18/v5n1a18.(dvi,ps,pdf)

Hiroo Shiga shiga@sci.u-ryukyu.ac.jp

Department of Mathematical Sciences, Colledge of Science,

Ryukyu University, Okinawa 903-0213, Japan

Toshihiro Yamaguchi tyamag@cc.kochi-u.ac.jp

Department of Mathematics Education,

Faculty of Education,

Kochi University,

Kochi 780-8520, Japan 